\title{
GENERATING CLASSES OF PERFECT BANACH SEQUENCE SPACES
}

\author{
G. CROFTS ${ }^{1}$
}

\begin{abstract}
A perfect sequence space $\lambda$ is said to be a step if $l^{1} \subset \lambda \subset l^{\infty}$ and $\lambda$ is a Banach space in its strong topology from $\lambda \times$. In this paper a method is given to generate additional steps from a step $\lambda$. Precisely, $\lambda^{p}$ is a step where $\lambda^{p} \equiv\left\{x=\left(x_{i}\right) \mid x_{i} \in C\right.$ and $\left.|x|^{\nu}=\left(\left|x_{i}\right|^{p}\right) \in \lambda\right\}$, for $1 \leqq p<\infty$, with norm $\|x\|_{\lambda^{p}}=\left(\left\||x|^{p}\right\|_{\lambda}\right)^{1 / p}$. It is shown that $\lambda^{p}, 1<p<\infty$, is reflexive iff $\lambda$ has a Schauder basis. The space of diagonal maps of $\lambda^{p}$ into $\lambda$ is characterized, as is the space of diagonal nuclear maps of $\lambda$ into $\lambda^{p}$ when $\lambda$ has a Schauder basis.
\end{abstract}

If $\lambda$ is a perfect sequence space which is a Banach space under the strong topology from $\lambda^{\times}$, and contains $l^{1}$ and is contained in $l^{\infty}$, we say that $\lambda$ is a step. Examples of steps in general include the Köthe dual of the usual sequence space associated with a Banach space possessing a normalized unconditional basis; see [4]. More specifically the $l^{p}$ spaces, the spaces $\mu_{a, p}$ and $v_{a, p}$ of Garling [5], and the spaces $m(\phi)$ and $n(\phi)$ of Sargent [13], are steps. In this paper we generate additional steps from a step $\lambda$ by paralleling a method of generating the $l^{p}$ spaces from $l^{1}$. In the cases where the usual coordinate vectors form a basis for $\lambda$, these generated spaces are reflexive. Others results paralleling the known properties of $l^{p}$ spaces are obtained under this additional hypothesis.

1. Definitions and preliminary results. The general terminology of this paper is as in [9]. Throughout we will assume that the sequence spaces $\lambda$ are normal and equipped with the topology $\mathscr{T}_{b}\left(\lambda^{\times}\right)$, unless we specifically state otherwise.

For sequences $x=\left(x_{i}\right), y=\left(y_{i}\right)$ we denote by $x y$ the sequence $\left(x_{i} y_{i}\right)$. Using the notation of Ruckle [12], we denote by $\mu^{j}$ the $\{u \mid u x \in \lambda$ for each

Presented to the Society, September 2, 1971 under the title Some properties of a special class of perfect Banach sequence spaces; received by the editors October 6, 1971 and, in revised form, March 10, 1972.

AMS 1970 subject classifications. Primary 46A45, 46B99.

Key words and phrases. Perfect sequence space, Banach space, regular sequence space, step, diagonal maps, multipliers of sequence spaces, nuclear maps, diagonal nuclear maps, reflexive.

${ }^{1}$ The author wishes to thank Professor J. R. Holub for enlightening discussions concerning ideas in this paper.

(c. American Mathematical Society 1972 
$x \in \mu\}$, where $\mu$ and $\lambda$ are sequence spaces. Since $\mu^{\lambda}$ is a normal sequence space, $\mu^{\lambda \lambda} \equiv\left(\mu^{\lambda}\right)^{\lambda}$ has meaning. If $\mu^{\lambda \lambda}=\mu$, we say that $\mu$ is $\lambda$-perfect. If $\lambda=l^{1}$, we use the standard terminology of [9] and write $\mu^{\times}$for $\mu^{\lambda}$ and say that $\mu$ is perfect if $\mu^{\times \times}=\mu$.

Let $x$ be the sequence $\left(x_{i}\right)$. We denote by $x^{(n)}$ the sequence $\left(y_{i}\right)$, where $y_{i}=x_{i}$ for $i \leqq n$, and $y_{i}=0$ for $i>n$.

Suppose $\lambda$ is a step and $u$ and $v$ elements in $\lambda$. We may choose the norm, $\|\cdot\|_{i}$, on $\lambda$ so that $\|u\|_{i} \leqq\|v\|_{\lambda}$ whenever $\left|u_{i}\right| \leqq\left|v_{i}\right|$ for each $i$; see [1]. Also, for $\lambda$ a step, $\lambda^{\times}$is a step (see [2]). We denote by $\lambda_{r}$ the $\left\{x \in \lambda \mid x^{(n)}\right.$ converges to $x$ in the $\mathscr{T}_{b}\left(\lambda^{\times}\right)$-topology\}. Under our general hypothesis it is easy to see that the topological dual of $\lambda_{r}$ is $\lambda^{\times}$. If $\lambda=\lambda_{r}$, we say that $\lambda$ is regular.

To eliminate some writing in the following sections we will always mean that $\lambda$ is a step when this symbol is used.

As a final preliminary we give the following parallel of the definition of $l^{p}$ :

Definition. For $\lambda$ a step and $1 \leqq p<\infty$, define $\lambda^{p}$ to be the $\left\{\left.x|| x\right|^{p} \equiv\right.$ $\left.\left(\left|x_{i}\right|^{p}\right) \in \lambda\right\}$.

2. Norming $\lambda^{p}$. For $x \in \lambda^{p}$ we let $\|x\|_{\lambda^{p}}$ denote the number $\left(\left\||x|^{p}\right\|_{\lambda}\right)^{1 / p}$. We show in this section that $\|\cdot\|_{\lambda^{p}}$ defines a norm on $\lambda^{p}$ which is strictly convex if $\lambda$ is regular. To simplify notation we let $\mu$ denote $\lambda^{p}$.

2.1 Minkowski inequality. If $x, y \in \mu$, then $\left\|x+y_{\mu} \leqq\right\| x\left\|_{\mu}+\right\| y \|_{\mu}$.

Proof. Let $x, y \in \mu$, and $u \in \lambda^{\times}$, with $\|u\|_{\lambda^{x}} \leqq 1$. By definition, $|x|^{p} \in \lambda$; so $\sum_{i=1}^{\infty}\left|u_{i}\right|\left|x_{i}\right|^{p}<\infty$ and $\left(|u|^{1 / p}\right)(x) \in l^{p}$. Thus $\left(|u|^{1 / p}\right)(x)$ and $\left(|u|^{1 / p}\right)(y)$ are in $l^{p}$. Using the familiar Minkowski inequality for the second inequality we have

$$
\begin{aligned}
\left(\left|\left\langle u,|x+y|^{p}\right\rangle\right|\right)^{1 / p} & \leqq\left(\sum_{i=1}^{\infty}\left(\left|u_{i}\right|^{1 / p}\left(\left|x_{i}\right|+\left|y_{i}\right|\right)\right)^{p}\right)^{1 / p} \\
& =\left(\sum_{i=1}^{\infty}\left(\left|u_{i}\right|^{1 / p}\left|x_{i}\right|+\left|u_{i}\right|^{1 / p}\left|y_{i}\right|\right)^{p}\right)^{1 / p} \\
& \leqq\left(\sum_{i=1}^{\infty}\left(\left|u_{i}\right|^{1 / p}\left|x_{i}\right|\right)^{p}\right)^{1 / p}+\left(\sum_{i=1}^{\infty}\left(\left|u_{i}\right|^{1 / p}\left|y_{i}\right|\right)^{p}\right)^{1 / p} \\
& =\left\langle|u|,|x|^{p}\right\rangle^{1 / p}+\left\langle|u|,|y|^{p}\right\rangle^{1 / p} \\
& \leqq\left(\left\||x|^{p}\right\|_{\lambda}\right)^{1 / p}+\left(\left\||y|^{p}\right\|_{\lambda}\right)^{1 / p} .
\end{aligned}
$$

The last inequality follows since $\|u\|_{j^{x}}=\|\mid u\|_{j^{x} \times}$. Hence

$$
\sup \left\{\left(\left|\left\langle u,|x+y|^{p}\right\rangle\right|\right)^{1 / p} \mid u \in \lambda^{\times},\|u\|_{\lambda^{x}} \leqq 1\right\} \leqq\|x\|_{\mu}+\|y\|_{\mu}
$$

and the conclusion holds.

Using 2.1 it is clear that $\left(\lambda^{p},\|\cdot\|_{\lambda^{\nu}}\right)$ is a normed space.

2.2 Proposition. The norm of $\mu$ is strictly convex if $\lambda$ is regular. 
ProOf. By way of contradiction assume the existence of a pair of elements $x, y$ in $\mu$ with $x \neq y,\|x\|_{\mu}=\|y\|_{\mu}=1$ and $\left\|\frac{1}{2}(x+y)\right\|_{\mu}=1$. Since $\lambda=\lambda_{r}$, we have $\lambda^{\prime}=\lambda^{\times}$, and hence there is an element $u$ in $\lambda^{\times}$with $\|u\|_{\lambda^{x}}=1$ and $\left\langle u,\left|\frac{1}{2}(x+y)\right|^{p}\right\rangle=1$. It is clear that $u$ may be chosen with $u_{i} \geqq 0$ for all $i$. Thus

$$
\begin{aligned}
1 & =\left(\sum_{i=1}^{\infty}\left(u_{i}^{1 / p}\left|\frac{x_{i}}{2}+\frac{y_{i}}{2}\right|\right)^{p}\right)^{1 / p}=\left(\sum_{i=1}^{\infty}\left|\left(u_{i}^{1 / p}\right)\left(\frac{x_{i}}{2}\right)+\left(u_{i}^{1 / p}\right)\left(\frac{y_{i}}{2}\right)\right|^{p}\right)^{1 / p} \\
& =\left\|\frac{1}{2}\left(\left(u^{1 / p}\right)(x)+\left(u^{1 / p}\right)(y)\right)\right\|_{p} .
\end{aligned}
$$

$l^{p}$ is strictly convex, so $\left\|\left(u^{1 / p}\right)(x)\right\|_{p}$ or $\left\|\left(u^{1 / p}\right)(y)\right\|_{p}$ is larger than 1. Assuming $\left\|\left(u^{1 / p}\right)(x)\right\|_{p}>1$ yields $\left\langle u,|x|^{p}\right\rangle>1$ and $\|x\|_{\mu}>1$.

3. $\lambda^{p}$ is a step which is $\lambda$-perfect. Again for simplicity of notation we let $\mu=\lambda^{p}$ and $\Psi=\lambda^{q}$, where $1 / p+1 / q=1$.

3.1 Hölder inequality. If $x \in \mu, z \in \Psi$, then $\|x z\|_{\lambda} \leqq\|x\|_{\mu}\|z\|_{\Psi}$.

Proof. For $u \in \lambda^{\times}, x \in \mu$, and $z \in \Psi$, it follows as in the proof of 2.1 that $\left(|u|^{1 / p}\right)(x) \in l^{p}$ and $\left(|u|^{1 / q}\right)(z) \in l^{q}$. Using the perfectness of $\lambda$, it clearly follows that $x z \in \lambda$.

Now assume $\|u\|_{\lambda \times} \leqq 1$. Using the standard Hölder inequality for the second inequality, we have

$$
\begin{aligned}
|\langle u, x z\rangle| & \leqq \sum_{i=1}^{\infty}\left|u_{i} x_{i} z_{i}\right| \leqq\left(\sum_{i=1}^{\infty}\left(\left|u_{i}\right|^{1 / p}\left|x_{i}\right|\right)^{p}\right)^{1 / p}\left(\sum_{i=1}^{\infty}\left(\left|u_{i}\right|^{1 / a}\left|z_{i}\right|\right)^{a}\right)^{1 / a} \\
& =\left\langle|u|,|x|^{p}\right\rangle^{1 / p}\left\langle|u|,|z|^{\alpha}\right\rangle^{1 / a} \leqq\|x\|_{\mu}\|z\|_{\Psi} .
\end{aligned}
$$

The conclusion follows as argued in 2.1 .

3.2 LEMMA. If $z \in \Psi$, then $\|z\|_{\Psi}$ equals the norm of $z$ as an operator from $\mu$ into $\lambda$.

Proof. By 3.1 we have, for $z \in \Psi$, that $z$ is an operator of $\mu$ into $\lambda$ and that the operator norm of $z$ is no larger than $\|z\|_{\Psi}$.

For the reverse inequality let $z \in \Psi$ and $\alpha=\left\||z|^{a}\right\|_{2}$. We have $\|z\|_{\Psi}=$ $(\alpha)^{1 / q}=(\alpha)\left(\alpha^{-1 / p}\right)=\left\||z|^{q} / \alpha^{1 / p}\right\|_{;}=\left\|(z)\left(|z|^{q / p}\right) / \alpha^{1 / p}\right\|_{\lambda} \leqq$ operator norm of $z$, since $\left\||z|^{q / p} / \alpha^{1 / p}\right\|_{\mu}=1$.

\subsection{Proposition. $\mu^{i}=\Psi^{\circ}$.}

Proof. In [1] it is shown that $\mu^{\lambda}$ is a step and that the norm of $\mu^{\lambda}$ is equivalent to the norm for operators of $\mu$ into $\lambda$. For $z \in \mu^{\lambda},\left|z_{i}^{(n)}\right| \leqq\left|z_{i}\right|$ for each $i$, so $\left\|z^{(n)}\right\|_{\mu^{\lambda}} \leqq\|z\|_{\mu^{i}}$ for each $n$. Thus the sequence $\left\{z^{(n)}\right\}$ is norm bounded in $\mu^{\lambda}$ and thus is a bounded sequence in the operator norm. Applying 3.2 and the fact that $\left\{z^{(n)}\right\} \subset \Psi^{\circ}$ we have $\left\{z^{(n)}\right\}$ a norm bounded sequence in $\Psi^{\cdot}$. Hence $\left\{\left|z^{(n)}\right|^{q}\right\}$ is a norm bounded sequence in $\lambda$ implying 
that $\left\{\left|z^{(n)}\right|^{q}\right\}$ is $\mathscr{T}_{s}\left(\lambda^{\times}, \lambda\right)$-bounded. Using the normality of $\lambda^{\times}$, the sequence $\left\{\sum_{i=1}^{n}\left|u_{i}\right|\left|z_{i}\right|^{q}\right\}$ is a bounded sequence of real numbers for each $u \in \lambda^{\times}$. Thus $\sum_{i=1}^{\infty}\left|u_{i}\right|\left|z_{i}\right|^{a}<\infty$ and $z \in \Psi^{\circ}$.

The reverse inclusion follows from 3.1.

3.4 THEOREM. $\lambda^{p}$ is a step which is $\lambda$-perfect.

Proof. 3.3 and the fact that $\Psi^{p}$ is a step show that $\lambda^{p}$ is a step. The $\lambda$-perfectness of $\lambda^{p}$ also follows from 3.3.

The following corollary parallels the result stating that $\left(l^{s}\right)^{t^{t}}=l^{r}$, where $1 / t=1 / r+1 / s ;$ see $[13]$ :

3.5 COROLLARY. $\left(\lambda^{s}\right)^{\lambda^{t}}=\lambda^{r}$, where $1 / t=1 / r+1 / s$.

PROOF. $\lambda^{s}=\left(\lambda^{t}\right)^{s / t}$ and $\lambda^{r}=\left(\lambda^{t}\right)^{r / t}$ with $t / s+t / r=1$. The result follows from 3.3 and 3.4.

The referee has observed the following generalized Hölder inequality:

3.6 Corollary. If $1 / t=1 / s+1 / r$ and $x \in \lambda^{s}, y \in \lambda^{r}$, then $x y \in \lambda^{t}$ with $\|x y\|_{\lambda^{t}} \leqq\|x\|_{\lambda^{s}}\|y\|_{\lambda \cdot \text {. }}$

ProOF. We need only check the norm inequality since the first conclusion is contained in 3.5. For $x \in \lambda^{s}, y \in \lambda^{r}$ it follows that $|x|^{t} \in \lambda^{s / t}$ and $|y|^{t} \in \lambda^{r / t}$. Using the definition of the $\lambda^{p}$ norm and 3.1 we have

$$
\begin{aligned}
\|x y\|_{\lambda^{t}} & =\left(\left\||x y|^{t}\right\|_{\lambda}\right)^{1 / t} \leqq\left[\left(\left\||x|^{t}\right\|_{\lambda^{s / t}}\right)\left(\left\||y|^{t}\right\|_{\lambda^{\prime r / t}}\right)\right]^{1 / t} \\
& =\left(\left(\left\||x|^{s}\right\|_{\lambda}\right)^{t / s}\right)^{1 / t}\left(\left(\left\||y|^{r}\right\|_{\lambda}\right)^{t / r}\right)^{1 / t}=\|x\|_{\lambda^{s}}\|y\|_{\lambda^{r} .}
\end{aligned}
$$

REMARKS. 1. Essentially repeating the proof that a sequence space $\nu=v^{\times}$if and only if $\nu=l^{2}$ (see [9]), we have $\nu=\nu^{i}$ if and only if $\nu=\lambda^{2}$.

Proof. One implication follows from 3.3.

Conversely, suppose $\nu=v^{\lambda}$, and let $x \in v$ with $\bar{x}=\left(\bar{x}_{i}\right)$. Then $x \bar{x}=$ $|x|^{2} \in \lambda$, so $\nu \subset \lambda^{2}$. However, this inclusion implies $\lambda^{2}=\left(\lambda^{2}\right)^{\lambda} \subset \nu^{\lambda}=v$.

2. In [3, §3], we find

THEOREM. If $\nu$ and $\zeta$ are arbitrary perfect sequence spaces and $\zeta$ is $\nu$ perfect, then each absolutely $\nu$-summing map is absolutely $\zeta$-summing.

It is observed in [3] that for $v=l^{t}, \zeta=l^{s}$, with $1 \leqq t \leqq s \leqq \infty$, the hypothesis of this theorem is satisfied. Corollary 3.5 above shows that for $\nu=\lambda^{t}$ and $\zeta=\lambda^{s}, 1 \leqq t \leqq s<\infty$, the above hypothesis is satisfied.

4. Diagonal nuclear maps and reflexivity of $\lambda^{p}$. The two main objectives of this section are to isolate the diagonal nuclear maps of $\lambda$ into $\lambda^{p}$ when $\lambda$ is regular, and show that $\lambda^{q}, 1<p<\infty$, is reflexive when $\lambda$ is regular.

4.1 Proposition. Each diagonal map of $\lambda^{p}$ into $\lambda^{s}, 1 \leqq s<p<\infty$, is compact if and only if $\lambda$ is regular. 
PROof. If $\lambda$ is regular, it clearly follows that $\lambda^{t}$ is regular, for $1 \leqq t<\infty$. In $\S 3$ of [1] it is shown that, for $v$ and $\zeta$ steps, the set of diagonal compact operators of $v$ into $\zeta$ is represented precisely by the set $\left(v^{\zeta}\right)_{r}$. The one implication now follows from 3.5.

Conversely, suppose $\lambda \neq \lambda_{r}$. There is then an $x$ in $\lambda, x=\left(x_{i}\right), x_{i} \geqq 0$, all $i$, with $x^{(n)}+x$ in the norm of $\lambda$. Then for some $\varepsilon_{0}>0$ we have $\left\|x-x^{(n)}\right\|_{i} \geqq$ $\varepsilon_{0}$, for all $n$. By definition $x^{1 / q} \in \lambda^{q}=\mu^{\lambda}$ and $\left\|x^{1 / q}-\left(x^{1 / q}\right)^{(n)}\right\|_{j q}=$ $\left(\left\|x-x^{(n)}\right\|_{\lambda}\right)^{1 / q} \geqq\left(\varepsilon_{0}\right)^{1 / q}$. Using 3.5 and the result from [1] used above we have the conclusion.

The following lemma is a slight modification of 3.1 of [8]. For the readers benefit we repeat the proof here using our notation.

4.2 Lemma. Let $v$ and $\zeta$ be steps both of which are regular. If all the diagonal maps from $v$ into $\zeta$ are compact, then there is a projection $P: K(\nu, \zeta) \rightarrow v^{\zeta}$ for which $\|P\|=1 .(K(\nu, \zeta)$ denotes the space of all compact maps of $v$ into $\zeta$ and $\nu^{\zeta}$ here means the space of diagonal matrices with diagonal from $v^{\zeta}$.)

Proof. Since $v=v_{r}$, all continuous linear maps of $v$ into $\zeta$ can be represented as matrices. Using the hypothesis that $\zeta=\zeta_{r}$, we have that $\zeta$ has a Schauder basis and hence, by $\left[14\right.$, p. 114], $v^{\prime} \approx \widetilde{\otimes} \zeta=K(\nu, \zeta)$.

Let $A=\left(a_{i j}\right) \in K(v, \zeta)$ and let $x \in \nu, u \in \zeta^{\times}$. $x u$, as a map of $\zeta$ into $v$, can be factored as $\zeta \rightarrow^{u} l^{1} \rightarrow^{i} c_{0} \rightarrow^{x} v$ where $i$ is the inclusion map of $l^{1}$ into $c_{0}$. It is known from [7] that $i$ is an integral map with norm $\leqq 1$. It is easy to see that the operator norms of $u$ and $x$ are respectively $\|u\|_{\zeta}$ and $\|x\|_{v}$. Thus $x u$ is an integral map of $\zeta$ into $v$ with integral norm less than $\|x\|_{v}\|n\|_{\zeta^{\times}}$(see [6]). Hence the diagonal matrix with diagonal $x u$ is a continuous linear form on $K(v, \zeta)$ [14, p. 168]. This means that $\sum_{i=1}^{\infty} a_{i i} x_{i} u_{i}<$ $\infty$, for each $x \in v, u \in \zeta^{\times} . v \zeta^{\times}$is normal by 1.1 of $[1]$; so $\sum_{i=1}^{\infty}\left|a_{i i} x_{i} u_{i}\right|<\infty$ for each $x u \in v \zeta^{\times}$. Thus $\left(a_{i i}\right) \in\left(\nu \zeta^{\times}\right)^{\times}=v^{\zeta}$ (see 1.2 of [1]) implying that the diagonal matrix with diagonal $\left(a_{i i}\right)$ is in $K(v, \zeta)$.

By 1.5 of [1], the norm of $v^{\zeta}$ can be chosen so that

$$
\begin{aligned}
\left\|\left(a_{i i}\right)\right\|_{v \zeta} & =\sup \left\{\mid \sum_{i=1}^{\infty} a_{i i} x_{i} u_{i} \|_{\left.\|x\|_{\nu} \leqq 1,\|u\|_{\zeta} \times \leqq 1\right\}}\right. \\
& \leqq \text { (integral norm of }(x u)) \text { (operator norm of } A) \\
& \leqq \text { (operator norm of } A),
\end{aligned}
$$

by the arguments above. Hence $P: K(v, \zeta) \rightarrow v^{\zeta}$ given by $P(A)=\left(a_{i i}\right)$ is the desired projection.

In the context of the above lemma the beneficial conclusion for us is that $v^{\zeta}$ has a topological complement in $K(v, \zeta)$, i.e. $K(v, \zeta)=v^{\prime} \approx \zeta=v^{\zeta} \oplus F$ for some subspace $F$ of $K(v, \zeta)$. The hypothesis of the lemma and the 
result from [1], given above in the proof of 4.1, yield $v^{\zeta}=\left(v^{5}\right)_{r}$. We now have $(K(v, \zeta))^{\prime}=J\left(\zeta, v^{\times}\right)=\left(v^{\zeta}\right)^{\prime} \oplus F^{\prime}=\left(v^{\zeta}\right)^{\prime} \oplus F^{\prime}$. Hence each element of $\left(v^{\zeta}\right)^{\times}$is an integral map of $\zeta$ into $v$ (see [6, p. 126]). Analyzing the identifications involved in the duality between $K(v, \zeta)$ and $J\left(\zeta, v^{\times}\right)$it can be seen that, for $v \in\left(v^{\zeta}\right)^{\times}$, the operation of $v$ as an integral map of $\zeta$ into $v$ is just coordinatewise multiplication.

We recall a theorem of Grothedieck [6, p. 134]:

THEOREM. Each integral linear map of a locally convex space $E$ into a Banach space $F$ is nuclear, if $F$ is separable and the strong dual of a Banach space.

Using this theorem we can now obtain a result similar to, but not the same as, 3.2 of [8].

4.3 Proposition. Let $\nu$ and $\zeta$ be steps which are regular and such that each diagonal map of $v$ into $\zeta$ is compact. Then a diagonal map $u$ of $\zeta$ into $v$ is nuclear if and only if $u \in\left(v^{5}\right)^{\times}$. In addition, the nuclear norm of $u$ is equivalent to the norm of $u$ in $\left(v^{\zeta}\right)^{\times}$, and $\left(v^{\zeta}\right)^{\times}$is regular.

Proof. Suppose $u \in\left(v^{5}\right)^{\times}$. From the above discussion we know that $u$ is an integral map of $\zeta$ into $v$. By hypothesis $\nu=v_{r}$ so $v$ is separable; $v$ is perfect, so $v=\left(\left(v^{\times}\right)_{r}\right)^{\prime}$. $u$ is a nuclear map of $\zeta$ into $v$ by the quoted theorem of Grothendieck.

Conversely, it is proved in [12] that the diagonal of $A$ is in $\left(\left(v^{5}\right)^{\times}\right)_{r}$, if $A$ is a nuclear matrix map of $\zeta$ into $v$. Hence $u \in\left(\left(\nu^{\zeta}\right)^{\times}\right)_{r} \subset\left(v^{\zeta}\right)^{\times}$whenever $u$ is a diagonal nuclear map of $\zeta$ into $v$.

It is proved in [6, p. 179] that the nuclear norm agrees with the dual norm from $K(\zeta, v)$. Thus by the above discussion the nuclear norm of $u \in\left(v^{\zeta}\right)^{\times}$is equivalent to $\|u\|_{(\nu \zeta)^{\times}}$.

The regularity of $\left(v^{5}\right)^{\times}$is clear from the earlier part of this proof. $\square$

The following corollary may be anticipated from Tong's result that the space of diagonal nuclear maps of $l^{p}$ into $l^{t}$, for $1 \leqq p<t<\infty$, is $l^{s}$, where $1 / p=1 / t+(s-1) / s ;$ see $[15]$ :

4.4 COROLlaRY. If $\lambda$ is regular, then the diagonal nuclear maps of $\lambda^{p}$ into $\lambda^{t}$, for $1 \leqq p<t<\infty$, are $\left(\lambda^{r}\right)^{\times}$, where $1 / p=1 / t+1 / r$.

Proof. Use 3.5 and 4.3.

REMARK. It is shown in 3.4 of [8] that for $t \leqq p$ the diagonal nuclear maps of $\lambda^{p}$ into $\lambda^{t}$ are just $l^{1}$.

4.5 Proposition. Let $v$ and $\zeta$ be steps which are regular. Then each diagonal map of $\nu$ into $\zeta$ is compact if and only if $\nu^{\zeta}$ is a reflexive space. 
Proof. If each diagonal map of $v$ into $\zeta$ is compact then $\left(v^{\zeta}\right)_{r}=v^{\zeta}$, so $\left(v^{5}\right)^{\prime}=\left(v^{5}\right)^{\times}$. By $4.3,\left(v^{5}\right)^{\times}=\left(\left(v^{5}\right)^{\times}\right)_{r}$, giving $\left(\left(v^{5}\right)^{\times}\right)^{\prime}=v^{\zeta}$.

Conversely, if $v^{\zeta}$ is reflexive, then $\left(\left(v^{\zeta}\right)^{\times}\right)_{r}$ is reflexive giving $\left(v^{\zeta}\right)^{\times}=$ $\left(\left(v^{\zeta}\right)^{\times}\right)_{r}$. Thus $\left(v^{\zeta}\right)^{\times}$is reflexive yielding $\left(v^{\zeta}\right)_{r}$ reflexive. Hence $\nu^{\zeta}=\left(v^{\zeta}\right)_{r}$, and each diagonal map of $v$ into $\zeta$ is compact.

4.6 COROLlary. For $1<p<\infty, \lambda^{p}$ is reflexive if and only if $\lambda$ is regular.

Proof. It is clear that $\lambda$ is regular if and only if $\lambda^{t}$ is regular, for $1 \leqq t<\infty$. The conclusion follows from 3.3 and 4.5 .

We conclude with the following problem:

If $\lambda$ is regular, are the diagonal $\lambda$-nuclear maps of $\lambda^{p}$ into $\lambda^{t}$, for $1 \leqq p<$ $t<\infty$, given by $\lambda^{s}$, where $1 / p=1 / t+(s-1) / s$ ? (See [10].)

\section{REFERENCES}

1. G. Crofts, Concerning perfect Fréchet spaces and diagonal transformations, Math. Ann. 182 (1969), 67-76. MR 40 \#3256.

2. E. Dubinsky, Perfect Fréchet spaces, Math. Ann. 174 (1967), 186-194. MR 36 \#3103.

3. E. Dubinsky and M. Ramanujan, Inclusion theorems for absolutely $\lambda$-summing maps, Math. Ann. 192 (1971), 177-190.

4. E. Dubinsky and J. R. Retherford, Schauder bases and Köthe sequence spaces, Trans. Amer. Math. Soc. 130 (1968), 265-280. MR 38 \#510.

5. D. J. H. Garling, A class of reflexive symmetric $B K$-spaces, Canad. J. Math. 21 (1969), 602-608.

6. A. Grothendieck, Produits tensoriels topologiques et espaces nucléaires, Mem. Amer. Math. Soc. No. 16 (1955). MR 17, 763.

7. - Sur certaines classes de suites dans les espaces de Banach, et le théorème de Dvoretzky-Rogers, Bol. Sci. Mat. São Paulo 8 (1953), 81-110. MR 20 \#1195.

8. J. R. Holub, Diagonal nuclear maps in sequence spaces, Math. Ann. 191 (1971), 326-332.

9. G. Köthe, Topological vector spaces. I, Die Grundlehren der math. Wissenschaften, Band 159, Springer-Verlag, New York, 1969. MR 40 \#1750.

10. M. S. Ramanujan, Generalized nuclear maps in normed linear spaces, J. Reine Angew. Math. 244 (1970), 190-197. MR 42 \#5016.

11. W. H. Ruckle, Diagonals of operators, Studia Math. 38 (1970), 43-49. MR 43 \#973.

12. - Topologies on sequence spaces, Pacific J. Math. (to appear).

13. W. L. C. Sargent, Some sequence spaces related to the $l^{p}$ spaces, J. London Math. Soc. 35 (1960), 161-171. MR 22 \#7001.

14. H. H. Schaefer, Topological vector spaces, Macmillan, New York, 1966. MR 33 \#1689.

15. A. Tong, Diagonal nuclear operators on $l^{p}$ spaces, Trans. Amer. Math. Soc. 143 (1969), 235-247. MR 40 \#4786.

Department of Mathematics, Virginia Polytechnic Institute and State UNIVERSITY, BLACKSBURG, VIRGINIA 24061 